\title{
Análise das Mudanças de Percepções de Estudantes de Engenharia de Computação após uma Oficina de Aprendizagem de Programação
}

\author{
Camille L. Jesús ${ }^{1}$, Roberto A. Bittencourt ${ }^{1}$ \\ ${ }^{1}$ UEFS - Universidade Estadual de Feira de Santana \\ Av. Transnordestina, s/n, Novo Horizonte \\ Feira de Santana - BA, Brasil - 44036-900 \\ camillejesus27@gmail.com, roberto@uefs.br
}

\begin{abstract}
Programming learning is a key piece in computing courses. However, programming is a difficult subject for students, especially for those who have never had any previous programming experience. Since 2013, the State University of Feira de Santana's Computer Engineering program has offered introductory programming workshops before the first academic term to reduce the initial difficulties faced by freshman students, and promote positive changes in their attitudes towards computing. This work aims to evaluate the attitude changes of the students who attended the workshop. Results suggest that students already had positive attitudes toward computing, and a significant change happened in their perceptions on the computing profession.
\end{abstract}

Resumo. A aprendizagem de programação é fundamental em cursos da área de computação. No entanto, programação é um assunto considerado difícil pelos estudantes, especialmente para os que nunca tiveram experiências anteriores com programação. Desde 2013, o curso de Engenharia de Computação da Universidade Estadual de Feira de Santana passou a oferecer oficinas de introdução à programação antes do semestre letivo, visando reduzir as dificuldades iniciais enfrentadas pelos estudantes do primeiro semestre e promover mudanças positivas nas suas atitudes em relação à computação. $O$ objetivo deste trabalho foi avaliar as mudanças de atitudes dos estudantes que participaram da oficina. Os resultados sugerem que as atitudes dos estudantes já eram positivas antes da oficina e que uma mudança significativa ocorreu nas percepções dos estudantes sobre a profissão de computação.

\section{Introdução}

A aprendizagem de programação é parte fundamental de cursos da área de computação. No entanto, é um assunto considerado difícil pelos estudantes, especialmente para os que nunca tiveram experiências anteriores com programação [Robins et al. 2003]. Existem diversas razões para estas dificuldades, as quais podem decorrer dos métodos de ensino utilizados pelos professores, das metodologias de estudo utilizadas pelos estudantes, das suas habilidades e fatores psicológicos, ou da própria natureza abstrata e complexa da programação [Lahtinen et al. 2005, Gomes and Mendes 2007].

Para aprender programação efetivamente, além de desenvolver habilidades específicas de programação, é necessário também possuir conhecimentos e habilidades 
VIII Congresso Brasileiro de Informática na Educação (CBIE 2019)

Anais dos Workshops do VIII Congresso Brasileiro de Informática na Educação (WCBIE 2019)

prévias, como a capacidade de resolução de problemas, raciocínio lógico, abstração, dentre outras [Jenkins 2002, Resnick et al. 2009]. Assim, não é difícil entender o alto índice de reprovação em disciplinas introdutórias de programação, também chamadas CS1. Pesquisas mostram que não houve grande melhoria na taxa de aprovação ao longo dos anos e, consequentemente, ainda há um número expressivo de reprovações [Watson and Li 2014, Bennedsen and Caspersen 2019].

Além das dificuldades citadas, os fatores psicológicos também podem afetar a aprendizagem. Os estudantes se deparam com a programação, uma novidade radical [Dijkstra et al. 1989], logo quando ingressam na universidade. Este período coincide com uma fase de transição e instabilidade na vida do estudante, seja pessoal ou financeira [Gomes and Mendes 2007]. Jenkins (2001) discorre sobre a motivação e o seu papel decisivo na permanência de um estudante em um curso. Hoegh e Moskal (2009) alertam que a prevalência de certas crenças ou percepções incorretas dos estudantes sobre a computação afetam os fatores motivacionais. Por isso, muitas vezes é importante mensurar as atitudes dos estudantes.

Desde 2013, o curso de Engenharia de Computação da Universidade Estadual de Feira de Santana (UEFS) passou a oferecer oficinas de introdução à programação antes do primeiro semestre letivo [Bittencourt et al. 2015], visando reduzir as dificuldades iniciais enfrentadas pelos estudantes do primeiro semestre e promover mudanças positivas nas suas atitudes em relação à computação.

O objetivo deste trabalho foi avaliar as mudanças de atitudes dos estudantes que passaram pela oficina. Com este propósito, foram levantadas as seguintes questões de pesquisa:

1. A oficina promoveu melhoria nas atitudes dos estudantes em relação à computação?

2. Quais as percepções dos estudantes sobre a abordagem e as ferramentas utilizadas na oficina?

\section{Fundamentação Teórica}

São descritos aqui os trabalhos prévios com abordagens CS0 e fundamentos sobre atitudes em relação à computação.

\subsection{Abordagens com CSO}

Uma das abordagens para ensino introdutório de programação é chamada de CS0. Ela consiste em um curso preliminar realizado antes do curso introdutório de programação (CS1) com o objetivo de atuar como ponte para este. Vihavainen et al. (2014), em seu estudo com diferentes abordagens, relatam que cursos CS0 foram um das abordagens com maior sucesso na melhoria da aprendizagem.

Kafura and Tatar (2001) relatam uma experiência inicial de um curso de pensamento computacional para promover uma compreensão precoce e profunda de conceitos primordiais da computação. O feedback dos estudantes sugere que o curso conseguiu ajudar na compreensão da natureza abstrata da computação e no desenvolvimento de modelos mentais, além de apresentar os conceitos mais importantes. 
VIII Congresso Brasileiro de Informática na Educação (CBIE 2019)

Anais dos Workshops do VIII Congresso Brasileiro de Informática na Educação (WCBIE 2019)

O trabalho de Wood e Clements (2018) traz o uso de diferentes abordagens de CS0 tais como robótica, jogos, música, arte computacional, segurança e aplicativos móveis levando a um impacto positivo sobre as taxas de aprovação dos estudantes, às suas atitudes em relação à computação e até à popularidade geral do curso de Ciência da Computação. Eles não verificaram diferenças significativas em relação às seis variações de metodologias utilizadas, ficando a critério do instrutor replicar livremente qualquer um dos tipos citados.

\subsection{Atitudes em Relação à Computação}

$\mathrm{Na}$ literatura, há uma ampla definição do conceito de atitudes. Num contexto mais específico, como a investigação da perspectiva de programadores iniciantes, LuxtonReilly et al. (2018) consideram atitudes como tendências psicológicas que incluem autopercepções, seja sobre si mesmos ou sobre características próprias específicas, podendo incluir até percepções de alguns aspectos de programação introdutória.

Aspectos como interesse, reconhecimento de competência e esforço refletem significativamente nas notas dos estudantes em disciplinas de programação introdutória [McKinney and Denton 2004]. Estes fatores afetivos diminuem ao longo do período de aprendizagem, um desafio que atualmente ainda persiste [Settle et al. 2015]. Influências atitudinais têm sido bastante associadas a medidas de sucesso no curso, especialmente autoeficácia e autopercepção [Kinnunen and Simon 2011, Kinnunen and Simon 2012]. Trabalhos recentes continuam destacando também diferenças na eficácia percebida entre os gêneros [Lishinski et al. 2016, Quille et al. 2017].

Pesquisas revelaram alguns fatores a serem superados para aumentar a participação dos estudantes, especialmente mulheres, em cursos de computação [Wiebe et al. 2003, Palaigeorgiou et al. 2005]. Hoegh e Moskal (2009) identificaram os seguintes problemas: i) falta de confiança em suas habilidades para aprender conceitos de ciência de computação; ii) a área é frequentemente associada como mais adequada a homens, desencorajando a participação feminina; iii) a vasta extensão das aplicações da computação é desconhecida pelos estudantes; iv) desestímulo devido aos estereótipos relacionados aos interessados na área; v) todos estas percepções incorretas combinadas geram pouco apelo à área tanto para homens quanto para mulheres.

Com o objetivo de mensurar a prevalência dessas percepções em relação à computação, Hoegh e Moskal (2009) desenvolveram e validaram um instrumento para levantamento de atitudes em relação à computação. $\mathrm{O}$ instrumento conta com um conjunto de 38 questões que abordam cada um dos fatores citados definidos em cinco construtos. São eles: Confiança, Gênero, Utilidade, Interesse e Profissional. O construto Confiança está relacionado à confiança dos estudantes em sua própria capacidade de aprender habilidades da computação; Gênero tem a ver com as percepções da área como sendo um campo preferencialmente masculino; Utilidade é a crença dos estudantes na serventia do conteúdo aprendido; Interesse é a motivação em relação à computação; e Profissional tem a ver com as crenças e perspectivas dos estudantes em relação à profissão da área de computação.

\section{Metodologia}

Esta seção descreve os métodos de pesquisa utilizados neste trabalho. São descritos o cenário, os participantes, a intervenção e os procedimentos de coleta e análise de dados. 
VIII Congresso Brasileiro de Informática na Educação (CBIE 2019)

Anais dos Workshops do VIII Congresso Brasileiro de Informática na Educação (WCBIE 2019)

\subsection{Cenário}

A presente pesquisa foi conduzida na Universidade Estadual de Feira de Santana (UEFS), instituição que oferece o curso de graduação em Engenharia de Computação.

\subsection{Participantes}

Os calouros do curso de Engenharia de Computação foram convidados a participar da oficina durante a matrícula na universidade, de modo que a participação foi voluntária. A oficina foi mediada por oito estudantes veteranos do curso: uma tutora e demais monitores. Contou com 26 participantes, dos quais 25 eram calouros e apenas um era veterano. Do total, 20 (76,92\%) eram do sexo masculino e seis (23,08\%), do sexo feminino. A idade média deles era de 18, $23 \pm 1,56$ anos. Apenas oito relataram ter algum conhecimento prévio de programação.

\subsection{A Intervenção}

A versão mais recente da oficina teve um relato de experiência descrito em detalhes em [Jesus et al. 2019]. Neste artigo de pesquisa, descrevemos sucintamente a intervenção. O primeiro dia começou com um tom introdutório, quando foram trabalhados conceitos de algoritmos, lógica de programação e fluxogramas através de atividades desplugadas. No segundo dia, propusemos a criação de uma animação livre e o desenvolvimento do jogo Pong em Scratch. No terceiro dia, continuamos trabalhando com a ferramenta Scratch no contexto da criação de jogos clássicos, como Interlagos e Arqueiro. Neste período, foram introduzidas estruturas de controle, variáveis e as interações entre objetos que a ferramenta proporciona (e.g., movimento, aparência, sensores). Nos dois últimos dias, o conhecimento adquirido até ali foi reforçado, fazendo a transição entre a linguagem visual Scratch para a linguagem textual Python. No quarto dia, foi utilizada a biblioteca Turtle de Python em atividades de desenho de figuras geométricas. No quinto dia, apenas com Python e entrada/saída pelo console, os estudantes desenvolveram os projetos de uma calculadora e de um jogo da adivinhação.

A oficina teve duração de 20 horas e contou com uma metodologia lúdica e baseada em aprendizagem ativa. Usamos ainda uma abordagem em espiral, com conceitos repetidos e em complexidade crescente [Bruner 1960]. Este tipo de abordagem permite a revisão dos conteúdos, o que contribui para a fixação do que foi abordado e oferece suporte para aprofundar as habilidades nos dias subsequentes. As explicações teóricas foram bastante breves e objetivas, sem muitos formalismos, com a maior parte do tempo sendo reservado para as atividades práticas. A participação dos estudantes era incentivada a todo momento pelos instrutores. Em caso de necessidade, os monitores estavam sempre disponíveis para auxiliarem.

\subsection{Coleta e Análise de Dados}

Para atender a questões éticas e preservar o anonimato dos participantes da pesquisa, um Termo de Consentimento Livre e Esclarecido (TCLE) foi disponibilizado e todos os participantes da oficina concordaram em participar e o assinaram. Os dados da oficina foram colhidos através da aplicação de dois questionários: o pré-intervenção; que tinha o intuito de obter alguns dados demográficos dos estudantes e suas atitudes preliminares em relação à computação, e o pós-intervenção; para mensurar novamente as atitudes e 
VIII Congresso Brasileiro de Informática na Educação (CBIE 2019)

Anais dos Workshops do VIII Congresso Brasileiro de Informática na Educação (WCBIE 2019)

também obter um feedback dos estudantes em relação à oficina. O questionário de atitudes aplicado foi uma tradução baseada no questionário para avaliação de atitudes e percepções em relação à computação de Hoegh e Moskal (2009) ${ }^{1}$.

Através dos dados quantitativos colhidos a partir dos questionários, foram calculadas estatísticas descritivas, como medidas de centralidade e dispersão, assim como tabelas de frequência, boxplots e gráficos de barras empilhadas. Inferências sobre as mudanças de atitudes após a intervenção foram computadas através do teste de hipóteses de Wilcoxon para dados não paramétricos.

\section{Resultados}

O questionário de atitudes é composto por 38 questões sobre cinco construtos: Confiança (8), Gênero (10), Utilidade (10), Interesse (6) e Profissional (4). Utilizamos uma escala de Likert de quatro níveis para as respostas às questões, sendo eles: Discordo Totalmente, Discordo Parcialmente, Concordo Parcialmente e Concordo Totalmente. Cada construto teve seu valor computado pela média das respostas de cada participante às questões do construto, sendo os valores da escala convertidos em valores numéricos de 1 a 4 .

Todos os conjuntos de dados, de cada categoria, foram testados quanto à normalidade pelo teste de Shapiro-Wilk, em que uma significância superior a 0,05 representa uma distribuição normal. Devido a não normalidade de algumas das distribuições padronizamos o uso de testes não paramétricos para todos os dados. Na Tabela 1, são descritos a mediana e o intervalo interquartis pré- e pós-oficina, além do tamanho da amostra, para cada construto.

Tabela 1. Resultados da análise descritiva.

\begin{tabular}{|c|l|l|c|}
\hline Atitude & $\begin{array}{l}\text { Pré-Oficina } \\
\text { Mediana (IIQ) }\end{array}$ & $\begin{array}{l}\text { Pós-Oficina } \\
\text { Mediana (IIQ) }\end{array}$ & $\mathbf{n}$ \\
\hline Confiança & $3,57(0,37)$ & $3,63(0,40)$ & 26 \\
\hline Interesse & $3,70(0,42)$ & $3,65(0,52)$ & 26 \\
\hline Gênero & $4,00(0,02)$ & $4,00(0,02)$ & 26 \\
\hline Utilidade & $3,83(0,42)$ & $3,75(0,83)$ & 26 \\
\hline Profissional & $2,75(0,31)$ & $3,25(1,00)$ & 26 \\
\hline
\end{tabular}

Como os conjuntos de dados comparados são provenientes dos mesmos participantes, foi realizado o teste não paramétrico de Wilcoxon para cada categoria, a fim de verificar se houve diferença significativa para cada atitude antes e após a intervenção. Na Tabela 2, encontram-se o escore-Z, a significância e o tamanho da amostra para cada atitude.

\footnotetext{
${ }^{1}$ O questionário utilizado consta em um site de suporte: https://sites.google.com/site/walgprog2019cs0/
} 
VIII Congresso Brasileiro de Informática na Educação (CBIE 2019)

Anais dos Workshops do VIII Congresso Brasileiro de Informática na Educação (WCBIE 2019)

Tabela 2. Resultados dos testes de Wilcoxon.

\begin{tabular}{|c|c|c|c|}
\hline Atitude & $\mathbf{Z}$ & Valor-p & $\mathbf{n}$ \\
\hline Confiança & $-0,46$ & 0,96 & 26 \\
\hline Interesse & $-0,37$ & 0,71 & 26 \\
\hline Gênero & $-0,64$ & 0,52 & 26 \\
\hline Utilidade & $-0,72$ & 0,47 & 26 \\
\hline Profissional & $-2,64$ & $\mathbf{0 , 0 1}$ & 26 \\
\hline
\end{tabular}

As mudanças de atitudes em relação à profissão apresentaram diferença significativa $(\mathrm{p} \leq 0,05)$ após a realização da oficina. Os box-plots dos resultados da categoria Profissional podem ser vistos na Figura 5. Os resultados pós-oficina apresentam uma dispersão maior. Entretanto, o construto pós-oficina possui uma mediana muito superior à do outro grupo e os dados estão dispersos em valores maiores.

Diante dos resultados obtidos, podemos observar que a mediana do fator Confiança sofreu um leve aumento, porém não houve ganho significativo para este construto. O seu diagrama pode ser visto na Figura 1. Os resultados para o fator Interesse demonstram que a mediana sofreu uma leve redução, agora os dados estão mais dispersos pelo terceiro quartil, como mostra a Figura 2. O fator Gênero possui a mediana pré e pós com o valor máximo e detém o maior número de outliers de todas as categorias. O diagrama para este construto pode ser visto na Figura 3. Os resultados para o fator Utilidade demonstram que a mediana sofreu uma leve redução e uma maior dispersão dos dados, como mostra a Figura 4.

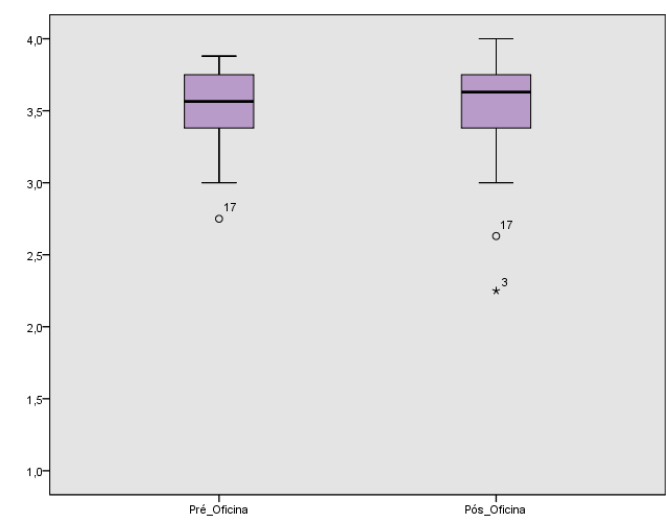

Figura 1. Construto Confiança.

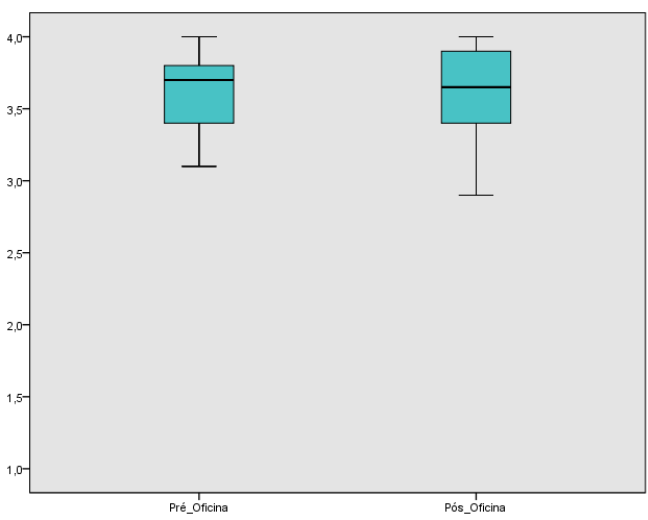

Figura 2. Construto Interesse.

A partir de dados do questionário pós-intervenção, mensuramos a opinião dos participantes sobre a oficina. Dos 26 participantes, apesar de quase metade deles julgarem a oficina como um pouco cansativa, $96 \%$ classificaram a oficina como muito estimulante e facilitadora do aprendizado e, mais de $88 \%$ acharam muito boa a didática utilizada e a experiência bastante proveitosa (Figura 6). Além disso, 80,8\% dos estudantes concordaram total ou parcialmente que a metodologia baseada em desafios práticos estimularam a aprendizagem, assim como $88,5 \%$ concordaram que a utilização de jogos motivou a aprendizagem e $92,3 \%$ consideraram a quantidade de monitores na oficina bastante ade- 
VIII Congresso Brasileiro de Informática na Educação (CBIE 2019)

Anais dos Workshops do VIII Congresso Brasileiro de Informática na Educação (WCBIE 2019)

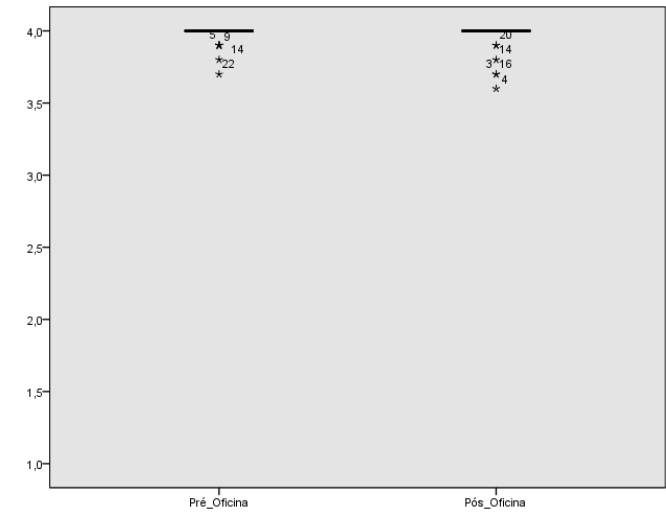

Figura 3. Construto Gênero.

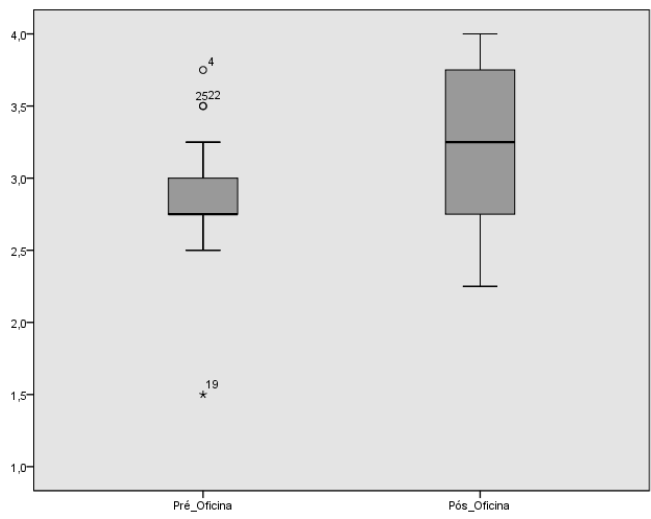

Figura 5. Construto Profissional.

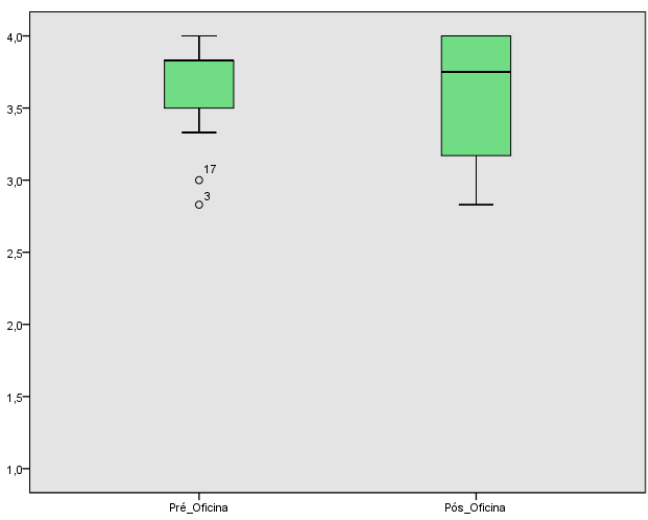

Figura 4. Construto Utilidade.

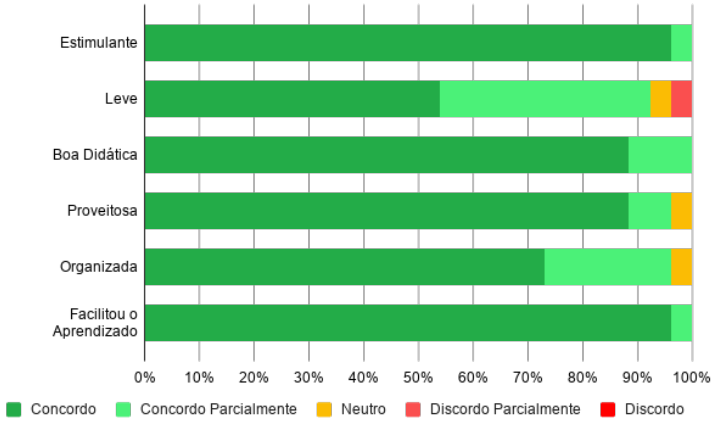

Figura 6. Opinião dos participantes sobre a oficina.

quada.

Em relação às ferramentas, Scratch obteve resultados positivos em mais de $80 \%$ (com concordância parcial ou total) para todos os aspectos de análise avaliados. Foram analisados aspectos como facilidade em usar, ambiente amigável, entre outros. A linguagem Python foi bem avaliada em promover a criatividade dos estudantes e o fator com avaliação menos positiva foi em relação ao ambiente de desenvolvimento utilizado, o IDLE, ser amigável, como pode ser visto na Figura 7.

\section{Discussão}

Neste trabalho, tentamos verificar o impacto da intervenção nas atitudes dos estudantes. Aqui tentamos fornecer respostas para nossas questões de pesquisa.

A primeira questão foi: A oficina promoveu melhoria nas atitudes dos estudantes em relação à computação? Para respondê-la, realizamos uma análise descritiva, através das medianas, e testes de hipótese para cada construto. Para facilitar a comparação, foram gerados gráficos de caixas para as distribuições dos valores pré e pós-oficina. Verificamos que houve diferença significativa apenas para o construto Profissional. Assim, não po- 

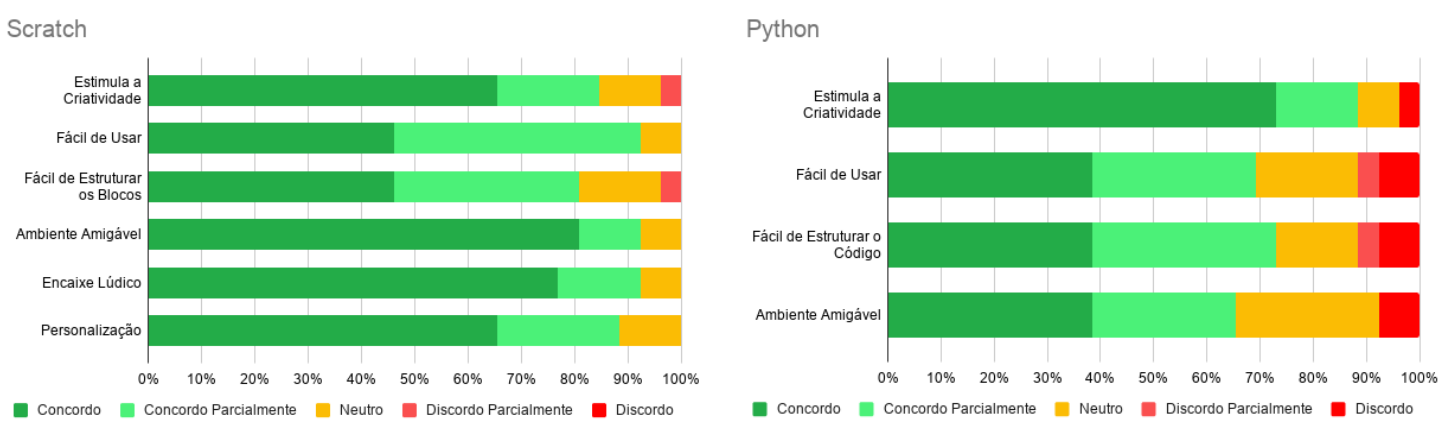

Figura 7. Opinião dos participantes sobre as ferramentas.

demos fazer conclusões acerca dos construtos Confiança, Interesse, Gênero e Utilidade. Porém, é importante ressaltar que os níveis preliminares já eram altos. Para ajudar a entender esta questão, utilizamos outros dados de percepção dos estudantes a partir do questionário pós-oficina.

Para Confiança, o pressuposto era que, após a oficina, os estudantes teriam adquirido conhecimento suficiente para resolver problemas simples e estariam mais confiantes em relação às suas habilidades em programação. Entretanto, descobrimos que esse fator praticamente se manteve inalterado, mesmo que mais de $88 \%$ dos estudantes concordaram que participar da oficina facilitou o aprendizado de programação de computadores. O mesmo ocorreu para Gênero, porém isso se deu ao fato de a grande maioria dos estudantes já terem percepções bastante positivas em relação ao sucesso tanto de homens quanto de mulheres na área de computação, conforme as questões específicas sobre este construto revelam. Outro resultado esperado era que a metodologia ativa e lúdica gerasse um aumento no Interesse dos estudantes pela computação, o que não é perceptível a partir dos resultados obtidos. Por fim, os resultados alcançados para Utilidade demostram que a oficina também não conseguiu aumentar a percepção de utilidade dos conteúdos que estavam sendo aprendidos em relação à área que eles pretendem seguir. O único construto que obteve resultados significativos foi o Profissional, com um aumento de quase $20 \%$. Isso pode se dar devido à experiência de aprendizagem ter proporcionado uma maior confiança dos estudantes em relação a profissões da área de computação, já que 73\% dos estudantes relataram que a oficina deu a eles uma boa noção de como funciona o curso de Engenharia de Computação.

A segunda questão de pesquisa foi: Quais as percepções dos estudantes sobre a abordagem e as ferramentas utilizadas na oficina? A partir de dados do questionário pós-intervenção, percebemos que a opinião dos participantes sobre a oficina foi bastante positiva sobre quase todos os aspectos (estímulo, didática, organização, proveito, aprendizado), sendo um pouco menos positiva por ser um pouco cansativa. Sobre as ferramentas, Scratch pontuou bem em todos os itens, enquanto que Python, apesar de possibilitar bastante a expressão da criatividade, foi um pouco pior em termos de usabilidade.

\section{Conclusões}

Este estudo teve o objetivo de analisar as atitudes dos estudantes de graduação em Engenharia de Computação durante uma oficina de aprendizagem de programação, buscando 
VIII Congresso Brasileiro de Informática na Educação (CBIE 2019)

Anais dos Workshops do VIII Congresso Brasileiro de Informática na Educação (WCBIE 2019)

entender melhor as suas percepções sobre a computação. Os resultados da pesquisa apontam que as atitudes em relação à profissão sofreram maior impacto durante a intervenção, enquanto que as outras atitudes não tiveram mudança significativa. A partir desses resultados, estratégias podem ser discutidas para abordar pontos específicos da oficina, tanto os aspectos positivos como as questões que precisam ser aprimoradas.

Embora os resultados não tenham sido os que esperávamos, eles nos permitiram levantar novas questões a serem pesquisadas, de forma exploratória. Por exemplo, como tornar os alunos mais confiantes de suas habilidades? Como a abordagem pode ser aprimorada para aumentar o interesse dos estudantes? Existe alguma diferença em quão rigorosos eles são na avaliação antes e após a experiência? Estas questões e diversas outras, assim como a motivação para facilitar o primeiro contato de estudantes com a programação, de maneira que não cause uma imagem ou percepção incorreta pelos estudantes, direcionam a necessidade de mais pesquisas. Pretendemos, em trabalhos futuros, mensurar as atitudes durante toda uma disciplina de CS1 e relacionar as atitudes mensuradas com o desempenho dos estudantes neste componente curricular.

\section{Agradecimentos}

Este projeto foi apoiado pela FAPESP através do auxílio de pesquisa 2015/24331-1 e da bolsa de treinamento técnico 201/14910-5.

\section{Referências}

Bennedsen, J. and Caspersen, M. E. (2019). Failure rates in introductory programming: 12 years later. ACM Inroads, 10(2):30-36.

Bittencourt, R. A., dos Santos, D. M. B., Rodrigues, C. A., Batista, W. P., and Chalegre, H. S. (2015). Learning programming with peer support, games, challenges and scratch. In 2015 IEEE Frontiers in Education Conference (FIE), pages 1-9. IEEE.

Bruner, J. S. (1960). The process of education. Harvard University Press.

Dijkstra, E. W. et al. (1989). On the cruelty of really teaching computing science. Communications of the ACM, 32(12):1398-1404.

Gomes, A. and Mendes, A. J. (2007). Learning to program-difficulties and solutions. In International Conference on Engineering Education-ICEE, volume 2007.

Hoegh, A. and Moskal, B. M. (2009). Examining science and engineering students' attitudes toward computer science. In 2009 39th IEEE Frontiers in Education Conference.

Jenkins, T. (2001). The motivation of students of programming. ACM SIGCSE Bulletin, 33(3):53-56.

Jenkins, T. (2002). On the difficulty of learning to program. In Proc. of the 3rd Annual Conference of the LTSN Centre for Information and Computer Sciences, volume 4.

Jesus, C. L., Santana, B. L., and Bittencourt, R. A. (2019). Oficinas de aprendizagem de programação com scratch e python em um curso de engenharia de computação. In WEI 2019 - XXVII Workshop sobre Educação em Computação.

Kafura, D. and Tatar, D. (2011). Initial experience with a computational thinking course for computer science students. In Proceedings of the 42nd ACM technical symposium on Computer science education, pages 251-256. ACM. 
VIII Congresso Brasileiro de Informática na Educação (CBIE 2019)

Anais dos Workshops do VIII Congresso Brasileiro de Informática na Educação (WCBIE 2019)

Kinnunen, P. and Simon, B. (2011). Cs majors' self-efficacy perceptions in cs1: results in light of social cognitive theory. In Proceedings of the seventh international workshop on Computing education research, pages 19-26. ACM.

Kinnunen, P. and Simon, B. (2012). My program is ok-am i? computing freshmen's experiences of doing programming assignments. Computer Science Education, 22(1):1-28.

Lahtinen, E., Ala-Mutka, K., and Järvinen, H.-M. (2005). A study of the difficulties of novice programmers. Acm Sigcse Bulletin, 37(3):14-18.

Lishinski, A., Yadav, A., Good, J., and Enbody, R. (2016). Learning to program: Gender differences and interactive effects of students' motivation, goals, and self-efficacy on performance. In Proceedings of the 2016 ACM Conference on International Computing Education Research, pages 211-220. ACM.

Luxton-Reilly, A., Albluwi, I., Becker, B. A., Giannakos, M., Kumar, A. N., Ott, L., Paterson, J., Scott, M. J., Sheard, J., Szabo, C., et al. (2018). Introductory programming: a systematic literature review. In Proc. Companion of the 23rd Annual ACM Conference on Innovation and Technology in Computer Science Education, pages 55-106.

McKinney, D. and Denton, L. F. (2004). Houston, we have a problem: there's a leak in the cs1 affective oxygen tank. ACM SIGCSE Bulletin, 36(1):236-239.

Palaigeorgiou, G., Siozos, P., Konstantakis, N. I., and Tsoukalas, I. A. (2005). A computer attitude scale for computer science freshmen and its educational implications. Journal of computer assisted learning, 21(5):330-342.

Quille, K., Culligan, N., and Bergin, S. (2017). Insights on gender differences in cs1: A multi-institutional, multi-variate study. In Proceedings of the 2017 acm conference on innovation and technology in computer science education, pages 263-268. ACM.

Resnick, M., Maloney, J., Monroy-Hernández, A., Rusk, N., Eastmond, E., Brennan, K., Millner, A., Rosenbaum, E., Silver, J. S., Silverman, B., et al. (2009). Scratch: Programming for all. Commun. Acm, 52(11):60-67.

Robins, A., Rountree, J., and Rountree, N. (2003). Learning and teaching programming: A review and discussion. Computer science education, 13(2):137-172.

Settle, A., Lalor, J., and Steinbach, T. (2015). Reconsidering the impact of cs1 on novice attitudes. In Proc. 46th ACM Technical Symposium on Computer Science Education.

Vihavainen, A., Airaksinen, J., and Watson, C. (2014). A systematic review of approaches for teaching introductory programming and their influence on success. In Proceedings of the tenth annual conference on International computing education research.

Watson, C. and Li, F. W. (2014). Failure rates in introductory programming revisited. In Proceedings of the 2014 conference on Innovation \& technology in computer science education, pages 39-44. ACM.

Wiebe, E., Williams, L., Yang, K., and Miller, C. (2003). Computer science attitude survey. computer science, 14(25):0-86.

Wood, Z. J., Clements, J., Peterson, Z., Janzen, D., Smith, H., Haungs, M., Workman, J., Bellardo, J., and DeBruhl, B. (2018). Mixed approaches to cs0: Exploring topic and pedagogy variance after six years of cs0. In Proceedings of the 49th ACM Technical Symposium on Computer Science Education, pages 20-25. ACM. 